\title{
The CORALS survey I: New estimates of the number density and gas content of damped Lyman alpha systems free from dust bias ${ }^{\star, \star \star}$
}

\author{
S. L. Ellison ${ }^{1}$, L. Yan ${ }^{2}$, I. M. Hook ${ }^{3}$, M. Pettini ${ }^{4}$, J. V. Wall ${ }^{5}$, and P. Shaver ${ }^{6}$ \\ 1 European Southern Observatory, Casilla 19001, Santiago 19, Chile \\ 2 SIRTF Science Center, Caltech, California, USA \\ e-mail: lyan@ipac.caltech.edu \\ 3 Astrophysics: Department of Physics, Nuclear and Astrophysics Laboratory, Keble Road, Oxford, OX1 3RH, UK \\ e-mail: ihook@gemini.edu \\ 4 Institute of Astronomy, Madingley Rd., Cambridge, CB3 0HA, UK \\ e-mail: pettini@ast.cam.ac.uk \\ 5 Astrophysics: Department of Physics, Nuclear and Astrophysics Laboratory, Keble Road, Oxford, OX1 3RH, UK \\ e-mail: jvw@astro.ox.ac.uk \\ 6 European Southern Observatory, Karl-Schwarzschild-Str. 2, 85748 Garching bei Munchen, Germany \\ e-mail: pshaver@eso.org
}

Received 19 June 2001 / Accepted 12 September 2001

\begin{abstract}
We present the first results from the Complete Optical and Radio Absorption Line System (CORALS) survey. We have compiled a homogeneous sample of radio-selected QSOs from the Parkes Catalogue and searched for damped Lyman alpha systems (DLAs) towards every target, irrespective of its optical magnitude. This approach circumvents selection effects - particularly from intervening dust - which have long been suspected to affect DLA surveys in optically-selected, magnitude-limited QSO samples. The CORALS data set consists of $66 z_{\mathrm{em}} \geq 2.2$ QSOs in which 22 DLAs with absorption redshifts $1.8 \leq z_{\mathrm{abs}} \leq z_{\mathrm{em}}$ have been identified over a total redshift interval $\Delta z=55.46$. Three of the DLAs are classified as "associated" systems with $z_{\mathrm{abs}} \sim z_{\mathrm{em}}$; of the 19 intervening DLAs, 17 are new discoveries. In this first paper of the CORALS series we describe the sample, present intermediate resolution spectroscopy and determine the population statistics of DLAs. We deduce a value of the neutral gas mass density traced by DLAs (expressed as a fraction of the closure density) $\log \Omega_{\text {DLA }} h=-2.59_{-0.24}^{+0.17}$, and a number density of DLAs per unit redshift $n(z)=0.31_{-0.08}^{+0.09}$, both at a mean redshift $\langle z\rangle=2.37$. Both values are only marginally higher than those measured in optically selected samples of QSOs. Taking into account the errors, we conclude that dust-induced bias in previous surveys may have led to an underestimate of these quantities by at most a factor of two. While $n(z)$ is greater in fainter $(B>20)$ QSOs, the effect is only at the $\sim 1 \sigma$ level and we have not uncovered a previously unrecognised population of high column density $\left(N(\mathrm{H} \mathrm{I})>10^{21} \mathrm{~cm}^{-2}\right)$ DLAs in front of faint QSOs. These conclusions are tentative because of the limited size of our data set; in particular the distribution of column densities is poorly sampled at the high end where a much larger survey of radio-selected QSOs is required the improve the statistics.
\end{abstract}

Key words. galaxies: quasars: absorption lines - ISM: dust, extinction - galaxies: evolution

\section{Introduction}

The presence of intervening gas in the sightline to a high redshift QSO will cause absorption lines to be superimposed on the quasar's continuum. This relatively

Send offprint requests to: S. Ellison, e-mail: sellison@eso.org

* The work presented here is based in part on data obtained with the ESO facilities on La Silla (EFOSC/3.6-m) and Paranal (FORS1/UT1).

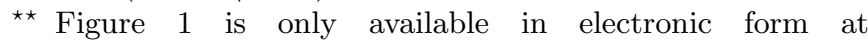
http://www.edpsciences.org simple physical process allows us to study in detail the properties of gas clouds in front of QSOs, whether in the intergalactic medium or in the galaxies giving rise to high column density damped Lyman alpha Systems (DLAs). DLAs are one of the key components of the universe at high redshift because, although relatively rare, they account for most of the neutral gas available for star formation.

Measuring the redshift evolution of $\Omega_{\mathrm{DLA}}$ (the total amount of neutral gas traced by DLAs expressed as a 
fraction of the closure density) has traditionally been seen as a tool for probing the history of assembling galaxies and measuring the rate at which they convert gas into stars (e.g. Lanzetta et al. 1995; Pei \& Fall 1995; StorrieLombardi et al. 1996; Storrie-Lombardi \& Wolfe 2000; Rao \& Turnshek 2000). Similarly, chemical abundances in DLAs have been used to trace the metallicity evolution of galaxies over most of the Hubble time (e.g. Pettini et al. 1997; Pettini et al. 1999; Prochaska \& Wolfe 1999, 2000). However, the interpretation of both DLA abundances and $\Omega_{\text {DLA }}$ as indicators of galaxy evolution is pivotal upon the assumption that DLAs are representative of the bulk of "normal" galaxies at each redshift sampled. Therefore, it is rather surprising that, at least for current samples of DLAs, no strong evolution of either $\Omega_{\mathrm{DLA}}$ or metallicity $\left(Z_{\mathrm{DLA}}\right)$ has been revealed by the above studies from $z \sim 3.5$ down to $z \sim 0.5$. One possible explanation is that DLAs are representative of only a particular evolutionary stage in a galaxy's lifetime. Alternatively, current DLA samples may be incomplete due to a selection bias. In this paper, we specifically investigate the possibility that dust extinction may have produced incomplete samples of DLAs in previous surveys based on optically-selected QSOs.

DLAs appear to remain relatively metal-poor $\left(Z_{\mathrm{DLA}} \approx\right.$ $\frac{1}{10}-\frac{1}{30} Z_{\odot}$ ) from $z \sim 3.5$ to $z \sim 0.5$ (Pettini et al. 1999 Prochaska \& Wolfe 2000). However, it has been noted by several authors that the absorbers with the highest values of hydrogen column density are all of low metallicity and dust content (e.g. Pettini et al. 1997; Boissé et al. 1998; Pettini et al. 1999; Prantzos \& Boissier 2000; Savaglio 2001). Whilst relatively metal-rich damped systems do exist, they tend to have values of $N(\mathrm{H} \mathrm{I})$ towards the lower end of the range considered for DLAs, $N(\mathrm{H} \mathrm{I}) \geq 2 \times 10^{20} \mathrm{~cm}^{-2}$. Boissé et al. (1998) and Prantzos $\&$ Boissier (2000) in particular have pointed out an apparent anti-correlation between $N(\mathrm{H} \mathrm{I})$ and metallicity. Since the census of metal abundances (using the column-density weighted average) is dominated by the highest $N(\mathrm{H} \mathrm{I})$ absorbers, such an anti-correlation could explain the lack of metallicity evolution in the present samples of DLAs. This anti-correlation between $N(\mathrm{H} \mathrm{I})$ and metallicity may reflect a bias against high-column-density, metal-rich DLAs with sufficient dust to obscure background QSOs from the eyes of magnitude-limited optical surveys.

Further evidence that optical magnitude-limited QSO samples may be incomplete due to dust bias comes from consideration of the spectral energy distribution of quasars with and without DLAs. Fall et al. (1989) and Pei et al. (1991) found evidence that QSOs with DLAs have systematically steeper continuum slopes than quasars with no damped absorber. A similar conclusion was reached by Carilli et al. (1998) who found that a high fraction of their "red" QSO sample had associated $21 \mathrm{~cm}$ absorbers compared with QSOs selected solely for the presence of Mg II absorbers. By modelling the extinction effect of dust, Pei $\&$ Fall (1995) estimated that at $z=3$ between $27 \%$ and
$44 \%$ of QSOs and $23 \%-38 \%$ of DLAs may be missing from $B$-band selected quasar samples.

The objective of the Complete Optical and Radio Absorption Line System (CORALS) survey presented here is simple and straightforward: compile a homogeneous sample of radio-selected QSOs and obtain medium resolution spectra of every target, regardless of optical magnitude. In this way it should be possible to determine quantitatively the severity of the dust bias implied by the observations reviewed above. The present paper is the first in the CORALS series. Here we define the sample (Sect. 3), describe the observations and present the QSO spectra (Sect. 4), identify the DLAs (Sect. 5), and calculate their number density and $\Omega_{\mathrm{DLA}}$ (Sect. 6).

We adopt an $\Omega_{M}=1.0, \Omega_{\Lambda}=0$ cosmology and use $H_{0}=65 \mathrm{~km} \mathrm{~s}^{-1} \mathrm{Mpc}^{-1}$ throughout the paper.

\section{Previous DLA surveys}

It is useful at this point to review some of the previous surveys for damped Lyman $\alpha$ systems and summarise their sample definitions and major findings. Relevant data are collected in Table 1.

The first major survey for DLAs was conducted by Wolfe et al. (1986) (WTSC86) from the relatively bright Lick QSO sample. The strategy adopted by those authors was to obtain low resolution spectra for a large number of QSOs in order to identify absorption systems above a certain equivalent width limit (in this case $W_{0} \geq 5 \AA$ ). These candidate DLAs were then followed-up with $2 \AA$ resolution spectroscopy revealing that approximately $50 \%$ were indeed damped, the remainder being blends of lower column density lines. This strategy of pre-selecting DLA candidates from low resolution spectra, based on restframe equivalent width, has come to characterise surveys in subsequent years. The approach allows a large sample of QSOs to be considered, maximising efficient use of observing time. The WTSC86 survey represents a landmark in absorption line studies, being the first major compilation of spectra and line lists for DLAs. One of the main results was that the incidence of DLAs was found to be greater than expected if the absorption were due to galaxies with the same cross section for H I absorption as present-day spirals (Wolfe 1988). In addition, the procedures for identifying DLAs were established and the $N(\mathrm{H} \mathrm{I})$ definition of a damped system set by this work, $N(\mathrm{H} \mathrm{I}) \geq 2 \times 10^{20} \mathrm{~cm}^{-2}$, has been upheld in all subsequent studies.

A survey of similar size to the WTSC86 sample was conducted a few years later by Sargent et al. (1989) (SSB89), although the initial focus of this work was on Lyman limit systems (LLSs). All of the quasars in the SSB89 sample were observed at a resolution of $4 \AA$, considerably higher than the first pass made by Wolfe et al. (1986). However, SSB89 estimate that their DLA sample is probably only complete for systems with $N(\mathrm{H} \mathrm{I}) \geq 10^{21} \mathrm{~cm}^{-2}$, although several lower column density lines were identified. Combining the SSB89 dataset with a similar number of QSOs found in the literature, 
Table 1. Summary of past surveys and their sample definitions. Column 3 tabulates the number of QSOs new to each survey. Therefore, if a particular survey has been supplemented with a previous sample, the total number of QSOs is the sum of the individual entries in Col. 3.

\begin{tabular}{|c|c|c|c|c|c|c|}
\hline Survey reference & Abbreviation & No. QSOs & $\begin{array}{c}\text { Supplemented } \\
\text { with }\end{array}$ & $\begin{array}{l}\text { Limiting Mag. } \\
\text { (Band) }\end{array}$ & Telescope & Resolution \\
\hline Wolfe et al. (1986) & WTSC86 & 68 & $\ldots$ & $18.5(V)$ & Lick & $10 \AA$ \\
\hline Sargent et al. (1989) & SSB89 & 53 & $\ldots$ & $N / A^{a}$ & Hale 5-m & $4 \AA$ \\
\hline Lanzetta et al. (1991) & LAN91 & 57 & SSB89 & $\begin{array}{l}\text { Literature } \\
\text { search }\end{array}$ & $\begin{array}{l}\text { Hale 5-m and Las } \\
\text { Campanas } 2.5-\mathrm{m}\end{array}$ & $4-6 \AA$ \\
\hline Lanzetta et al. (1995) & LWT95 & 260 & LAN91 & $20(V)^{b}$ & IUE & $7-10 \AA$ \\
\hline Wolfe et al. (1995) & WLFC95 & 228 & LWT95 & $18.75(B)$ & $\begin{array}{l}\text { MMT and Las } \\
\text { Campanas 2.5-m }\end{array}$ & $6-10 \AA$ \\
\hline $\begin{array}{l}\text { Storrie-Lombardi } \\
\& \text { Wolfe }(2000)\end{array}$ & SLW00 & 40 & $\begin{array}{l}\text { Many incl. WTSC86, } \\
\text { LWT95, WLFC95 }\end{array}$ & $19.5(R)^{b}$ & $\begin{array}{c}\text { Keck, AAT } \\
\text { Lick }\end{array}$ & $2 \AA$ \\
\hline Péroux et al. (2001a) & PER01 & 66 & SLW00 & $20.5(R)^{b}$ & $\begin{array}{l}\text { 4-m CTIO } \\
\text { WHT }\end{array}$ & $5 \AA$ \\
\hline
\end{tabular}

Notes: ${ }^{a}$ No broad-band limit is quoted for SSB89, only a derived apparent magnitude from the continuum flux at $\lambda_{0}=1450 \AA$.

${ }^{b}$ This is the faintest magnitude in this sample, but it does not indicate a completeness limit.

Lanzetta et al. (1991) adopted the strategy of WTSC86 in making a $5 \AA$ equivalent width cut to select 89 DLA candidates from their sample of 101 QSOs.

From follow-up spectroscopy at $2-3 \AA$ resolution of these candidates, Lanzetta et al. (1991) produced the first thorough statistical analysis of damped system number density, the mass density of neutral gas in DLAs and their clustering properties. A few years later, Lanzetta et al. (1995) extended their work to include DLAs at lower redshift by exploiting ultraviolet data obtained from the International Ultraviolet Explorer (IUE) satellite to gain one of the first glimpses into the $z_{\text {abs }}<1.6$ absorber population. These authors found that $\Omega_{\text {DLA }}$ decreases significantly from $z \sim 3.5$ to $z \sim 0.01$, and interpreted this redshift evolution as evidence for the consumption of $\mathrm{H} \mathrm{I}$ gas by star formation.

Wolfe et al. (1995) (WLFC95) used the Large Bright Quasar Survey (LBQS, Hewett et al. 1995 and references therein) to search for DLAs with $1.6 \lesssim z_{\text {abs }} \lesssim 3.0$. A total of 59 DLA candidates out of 228 spectra were preselected as having $W_{0} \geq 5 \AA$. At the time of publishing their paper, Wolfe et al. (1995) had confirmed the identification of 13 DLAs with $N(\mathrm{H} \mathrm{I}) \geq 2 \times 10^{20} \mathrm{~cm}^{-2}$ from 15 candidates with $W_{0}>9 \AA$. In addition, there were 8 DLA candidates whose equivalent widths exceeded $10 \AA$ and were therefore considered highly likely to be damped systems. All of these 8 candidates have subsequently been confirmed as DLAs with intermediate resolution spectroscopy (Storrie-Lombardi \& Wolfe 2000). In addition to the LBQS sample, WLFC95 constructed a "statistical sample" from the literature consisting of 80 DLAs. From this they confirmed the coincidence which had previously been noted (e.g. Lanzetta 1993) that the mass of $\mathrm{H}$ I in DLAs at $z \sim 3$ is similar to the total luminous mass in stars today. Expressing both as fractions of the closure density, $\Omega_{\mathrm{DLA}}(z=3.25)=(5.1 \pm 1.7) \times$ $10^{-3} h_{50}^{-1}$ and $\Omega_{\star}(z=0) \simeq 5 \times 10^{-3} h_{50}^{-1}$ (e.g. Pagel 2000).

In order to extend studies of DLAs to lower redshifts, Rao \& Turnshek (2000) have recently published results from an observing campaign with the Hubble Space Telescope $(H S T)$. Space-based telescopes are required in order to detect low $z$ DLAs since the Lyman $\alpha$ signature at $z_{\text {abs }} \lesssim 1.6$ falls below the atmospheric cut-off. Since such satellite resources are limited, a pre-selection based on Mg II and Fe II line strengths determined from groundbased observations has permitted an efficient screening of the initial QSO sample. A total of 12 DLAs with $z_{\text {abs }}<1.5$ was found, thereby significantly improving the statistics in this redshift regime. The puzzling result obtained from these new data is that, contrary to previous indications, $\Omega_{\text {DLA }}$ appears to remain approximately constant over all redshifts sampled, from $z=3.5$ to 0.1. Using HST archival spectra, Churchill (2001) has also argued that the number density of DLAs implied from the incidence of $\mathrm{Mg}$ II systems remains unaltered down to $z_{\text {abs }} \sim 0.05$.

Two recent surveys have pushed the bounds to higher redshifts. Storrie-Lombardi \& Wolfe (2000) and Péroux et al. (2001a) have recently identified 19 and 15 DLAs respectively at $z_{\text {abs }}>3.5$, including the highest redshift damped absorber known to date $\left(z_{\mathrm{abs}}=4.466\right.$, Péroux et al. 2001a; Dessauges-Zavadsky et al. 2001). Again, there is only marginal evidence for evolution; between $z=3.5$ and $4.5 \Omega_{\mathrm{DLA}}$ is lower than at more recent epochs, but the effect is only at the $\sim 2 \sigma$ level.

The intention of this (non-exhaustive) review of previous DLA surveys is to illustrate the difficulty of 
extracting unbiased statistics from samples based on inhomogeneous datasets. Many of these surveys have built on existing samples, taken additional targets from the literature and sometimes have insufficient resolution to reliably determine whether an absorber is damped. The advantage of this approach has been largely a statistical one. The aggregate samples often contain several hundred QSOs and such a large number is indeed required in order to determine meaningful statistics because of the rarity of damped systems. The purpose of CORALS is to provide the first complete and homogeneous survey for DLAs free from any bias that may be inherent in magnitude-limited QSO samples.

\section{The CORALS survey sample}

We have based our new survey for DLAs on the radioselected Parkes quarter-Jansky sample (Shaver et al. 1996; Jackson et al. in prep.; Hook et al. in prep.). This complete sample of flat-spectrum radio sources was taken from the Parkes Catalogue (Wright \& Otrupcek 1990), which consists of radio and optical data for almost 8300 radio sources for the sky south of declination $+27 \mathrm{deg}$. Over most of its area, it is complete to $S_{2.7} \mathrm{GHz}=0.25 \mathrm{Jy}$. The Parkes quarter-Jansky sample consists of all (878 in total) flat-spectrum $(\alpha>-0.4$, measured at 2.7 and $5.0 \mathrm{GHz})$ sources with declinations between $+2.5^{\circ}$ and $-80^{\circ}$, excluding low galactic latitudes $\left(|b|<10^{\circ}\right)$ and regions around the Magellanic Clouds. The sample is complete to $S_{2.7} \mathrm{GHz}=0.25 \mathrm{Jy}$ over $4.39 \mathrm{sr}$ and to $0.60 \mathrm{Jy}$ over a further $1.16 \mathrm{sr}$.

Using this Parkes compilation of radio sources as the parent sample, optical identification and $B$-band magnitudes for all targets with $\delta>-45^{\circ}$ were achieved using the COSMOS Southern Sky Catalogue (Drinkwater et al. 1995) in the first instance or imaging at the ESO 3.6-m telescope at La Silla for the faintest QSOs. Therefore, optical counterparts were identified for every radio source in the Parkes compilation. From this complete sample of 878 sources with no optical magnitude limit or other selection bias, low resolution spectra $(F W H M=12-14 \AA)$ were obtained for the 442 stellar identifications (QSOs and BL Lacs) with the EFOSC on the ESO 3.6-m to determine redshifts. We note that since compiling the CORALS sample, the catalogue of PKS sources has been revised slightly to include some extra targets (Jackson et al. in prep.; Hook et al. in prep.). However, it is important to stress that this will not affect the results presented here, since the important factor in our sample definition is that it is optically complete.

In our ground-based search for DLAs in this sample we restricted ourselves to QSOs with $z_{\mathrm{em}} \geq 2.2$, so as to be able to record a sufficient portion of the spectrum blueward of the Ly $\alpha$ emission down to the onset of the atmospheric cut-off. This left us with a final sample of 66 QSOs in which we could search for DLAs in the range
Table 2. Summary of observations CORALS survey. Many of the bright targets were observed first with low resolution at the ESO 3.6-m and QSOs with strong absorbers followed-up with AAT observations, whilst others were observed directly with the AAT. All faint targets $(B>20)$ were observed only with the VLT.

\begin{tabular}{llcc}
\hline \hline Telescope & $\begin{array}{l}\text { Date } \\
\text { (no. nights) }\end{array}$ & $\begin{array}{c}\text { Resolution } \\
\text { (at 4000 } \AA)\end{array}$ & $\begin{array}{c}\text { No. of } \\
\text { QSOs observed }\end{array}$ \\
\hline ESO 3.6-m & Sep. 1998 (2) & $6-8 \AA$ & 16 \\
ESO 3.6-m & Feb. 1999 (2) & $7 \AA$ & 12 \\
AAT & Dec. 1998 $\left(3^{a}\right)$ & $3 \AA$ & 11 \\
AAT & Apr. 1999 $\left(2.5^{b}\right)$ & $3 \AA$ & 10 \\
AAT & Oct. 1999 $(2)$ & $3 \AA$ & 12 \\
VLT & Oct. 2000 $\left(0.5^{c}\right)$ & $4.5 \AA$ & 9 \\
VLT & Mar. 2001 $(2)$ & $4.5 \AA$ & 10 \\
VLT & June 2001 $\left(0.5^{d}\right)$ & $4.5 \AA$ & 1 \\
\hline
\end{tabular}

Notes: ${ }^{a}$ One night lost to bad weather.

${ }^{b}$ The April AAT run consisted of 5 half nights.

${ }^{c} 6$ hours of service time.

${ }^{d} 6$ hours of Director's discretionary time.

$1.8<z_{\text {abs }}<z_{\text {em }}$ with sufficient sensitivity to measure reliable values of the column density $N(\mathrm{H} \mathrm{I})^{1}$.

\section{Observations and spectral analysis}

Before commencing our observing campaign, we first searched the literature to determine which QSOs had already been observed at sufficiently high spectral resolution, $S / N$ and over the correct wavelength range to match the rest of our DLA survey. The eight QSOs for which adequate spectra were found in the literature were not re-observed. The remaining 58 QSOs were subject to an extensive observing campaign that spanned five semesters on three different telescopes; relevant details are presented in Table 2. References for the eight QSO spectra observed by others are given in Table 3 .

Our observing strategy has been to obtain "snapshot" spectra of as many bright (typically $B<20$ ) QSOs as possible at low resolution with the ESO 3.6-m in order to pre-select targets with candidate DLAs for higher resolution follow-up at the AAT. Targets fainter than this limit were observed directly with the VLT. We briefly review the main characteristics of the observations.

\subsection{ESO 3.6- $m$ observations}

We obtained low resolution spectra of 28 QSOs using the EFOSC2 spectrograph on the ESO 3.6-m telescope during four nights between Sep. 1998 and Feb. 1999. The spectral resolution is approximately $7 \AA$ with a 1 arcsec slit and B600 grism, covering 3270-5240 ̊. For a few QSOs,

\footnotetext{
${ }^{1}$ One of the observed QSOs actually has $z_{\mathrm{em}}<2.2$ due to an incorrect redshift in our original list, see Table 3 .
} 
Table 3. Complete list of all CORALS QSOs and the tally of DLAs identified. References refer to QSOs for which optical spectroscopy had already been obtained prior to our survey, as follows: 1 - Lanzetta et al. (1991); 2 - Pettini et al. (1997); 3 - Ledoux et al. (1998); 4 - Lu et al. (1996); 5 - M. Giavalisco, private communication; 6 - Tytler et al. (1996); 7 - Songaila (1998); 8 - Turnshek et al. (1989).

\begin{tabular}{|c|c|c|c|c|c|c|}
\hline QSO & $z_{\mathrm{em}}$ & $B$ mag & DLA? & $N(\mathrm{H} \mathrm{I})\left(10^{20} \mathrm{~cm}^{-2}\right)$ & $z_{\mathrm{abs}}$ & Ref \\
\hline B0017-307 & 2.666 & 19.0 & $\mathrm{~N}$ & $\ldots$ & $\ldots$ & \\
\hline B0039-407 & 2.478 & 18.5 & $\mathrm{~N}$ & $\ldots$ & $\ldots$ & \\
\hline B0104-275 & 2.492 & 18.5 & $\mathrm{~N}$ & $\ldots$ & $\ldots$ & \\
\hline B0113-283 & 2.555 & 19.0 & $\mathrm{~N}$ & $\ldots$ & $\ldots$ & \\
\hline B0122-005 & 2.280 & 18.5 & $\mathrm{~N}$ & $\ldots$ & $\ldots$ & \\
\hline B0244-128 & 2.201 & 18.5 & $\mathrm{~N}$ & $\ldots$ & $\ldots$ & \\
\hline B0256-393 & 3.449 & 19.6 & $\mathrm{~N}$ & $\ldots$ & $\ldots$ & \\
\hline B0325-222 & 2.220 & 19.0 & $\mathrm{~N}$ & $\ldots$ & $\ldots$ & \\
\hline B0329-255 & 2.685 & 17.1 & $\mathrm{~N}$ & $\ldots$ & $\ldots$ & 1 \\
\hline B0335-122 & 3.442 & 21.5 & $\mathrm{Y}$ & 6.0 & 3.178 & \\
\hline B0347-211 & 2.944 & 21.1 & $\mathrm{Y}$ & 2.0 & 1.947 & \\
\hline B0405-331 & 2.570 & 19.0 & $\mathrm{Y}$ & 4.0 & $2.570^{a}$ & \\
\hline В0420+022 & 2.277 & 19.5 & $\mathrm{~N}$ & $\ldots$ & $\ldots$ & \\
\hline B0422-389 & 2.346 & 18.0 & $\mathrm{~N}$ & $\ldots$ & $\ldots$ & \\
\hline B0432-440 & 2.649 & 19.6 & $\mathrm{Y}$ & 6.0 & 2.297 & \\
\hline B0434-188 & 2.702 & 20.0 & $\mathrm{~N}$ & $\ldots$ & $\ldots$ & \\
\hline B0438-436 & 2.863 & 19.5 & $\mathrm{Y}$ & 6.0 & 2.347 & \\
\hline B0451-282 & 2.560 & 19.0 & $\mathrm{~N}$ & $\ldots$ & $\ldots$ & \\
\hline B0458-020 & 2.286 & 20.0 & $\mathrm{Y}$ & 45.0 & 2.039 & 2 \\
\hline \multirow[t]{2}{*}{ B0528-250 } & 2.765 & 19.0 & $\mathrm{Y}$ & 5.6 & 2.141 & 3 \\
\hline & & & $\mathrm{Y}$ & 15.8 & $2.811^{a}$ & 4 \\
\hline B0537-286 & 3.110 & 20.0 & $\mathrm{Y}$ & 2.0 & 2.974 & \\
\hline B0601-172 & 2.711 & 20.0 & $\mathrm{~N}$ & $\ldots$ & $\ldots$ & \\
\hline B0610-436 & 3.461 & 19.0 & $\mathrm{~N}$ & $\ldots$ & $\ldots$ & 5 \\
\hline B0819-032 & 2.352 & 18.2 & $\mathrm{~N}$ & $\ldots$ & $\ldots$ & \\
\hline B0834-201 & 2.752 & 19.0 & $\mathrm{~N}$ & $\ldots$ & $\ldots$ & \\
\hline B0913+003 & 3.074 & 21.7 & $\mathrm{Y}$ & 5.5 & 2.744 & \\
\hline B0919-260 & 2.300 & 19.0 & $\mathrm{~N}$ & $\ldots$ & $\ldots$ & \\
\hline B0933-333 & 2.906 & 20.0 & $\mathrm{Y}$ & 3.0 & 2.682 & \\
\hline B1010-427 & 2.954 & 17.5 & $\mathrm{~N}$ & $\ldots$ & $\ldots$ & \\
\hline B1055-301 & 2.523 & 19.5 & $\mathrm{Y}$ & 35.0 & 1.904 & \\
\hline B1136-156 & 2.625 & 20.0 & $\mathrm{~N}$ & $\ldots$ & $\ldots$ & \\
\hline B1147-192 & 2.489 & 19.4 & $\mathrm{~N}$ & $\ldots$ & $\ldots$ & \\
\hline B1149-084 & 2.370 & 18.5 & $\mathrm{~N}$ & $\ldots$ & $\ldots$ & \\
\hline B1228-113 & 3.528 & 22.0 & $\mathrm{Y}$ & 4.0 & 2.193 & \\
\hline B1228-310 & 2.276 & 19.0 & $\mathrm{~N}$ & $\ldots$ & $\ldots$ & \\
\hline B1230-101 & 2.394 & 19.8 & $\mathrm{Y}$ & 3.0 & 1.931 & \\
\hline \multirow[t]{2}{*}{ B1251-407 } & 4.464 & 23.7 & $\mathrm{Y}$ & 4.0 & 3.533 & \\
\hline & & & $\mathrm{Y}$ & 2.0 & 3.752 & \\
\hline B1256-243 & 2.263 & 19.5 & $\mathrm{~N}$ & $\ldots$ & $\ldots$ & \\
\hline $\mathrm{B} 1318-263^{b}$ & 2.027 & 20.4 & $\mathrm{~N}$ & $\ldots$ & $\ldots$ & \\
\hline B1351-018 & 3.710 & 20.9 & $\mathrm{~N}$ & $\ldots$ & $\ldots$ & \\
\hline \multirow[t]{2}{*}{ B1354-107 } & 3.006 & 19.2 & $\mathrm{Y}$ & 2.5 & 2.501 & \\
\hline & & & $\mathrm{Y}$ & 6.0 & $2.966^{a}$ & \\
\hline B1402-012 & 2.518 & 18.2 & $\mathrm{~N}$ & $\ldots$ & $\ldots$ & \\
\hline B1406-267 & 2.430 & 21.8 & $\mathrm{~N}$ & $\ldots$ & $\ldots$ & \\
\hline B1418-064 & 3.689 & 18.5 & $\mathrm{Y}$ & 2.5 & 3.449 & \\
\hline B1430-178 & 2.331 & 19.0 & $\mathrm{~N}$ & $\ldots$ & $\ldots$ & \\
\hline B1535+004 & 3.497 & 24.1 & $\mathrm{~N}$ & $\ldots$ & $\ldots$ & \\
\hline B1556-245 & 2.813 & 18.5 & $\mathrm{~N}$ & $\ldots$ & $\ldots$ & \\
\hline B1635-035 & 2.871 & 21.8 & $\mathrm{~N}$ & $\ldots$ & $\ldots$ & \\
\hline
\end{tabular}

Notes: ${ }^{a} z_{\mathrm{abs}} \sim z_{\mathrm{em}} ;{ }^{b}$ The original redshift of this target was incorrect in our list, the VLT spectrum confirms the true redshift which is less than the survey cut-off at $z_{e m}=2.2$. 
Table 3. continued.

\begin{tabular}{|c|c|c|c|c|c|c|}
\hline QSO & $z_{\mathrm{em}}$ & $B$ mag & DLA? & $N(\mathrm{H} \mathrm{I})\left(10^{20} \mathrm{~cm}^{-2}\right)$ & $z_{\mathrm{abs}}$ & Ref \\
\hline $\mathrm{B} 1701+016$ & 2.842 & 21.7 & $\mathrm{~N}$ & $\ldots$ & $\ldots$ & \\
\hline B1705+018 & 2.575 & 18.9 & $\mathrm{~N}$ & $\ldots$ & $\ldots$ & \\
\hline B1937-101 & 3.780 & 19.0 & $\mathrm{~N}$ & $\ldots$ & $\ldots$ & 6 \\
\hline B2000-330 & 3.780 & 18.5 & $\mathrm{~N}$ & $\ldots$ & $\ldots$ & 7 \\
\hline B2126-158 & 3.275 & 17.5 & $\mathrm{~N}$ & $\ldots$ & $\ldots$ & 7 \\
\hline B2149-307 & 2.330 & 17.5 & $\mathrm{~N}$ & $\ldots$ & $\ldots$ & \\
\hline B2212-299 & 2.703 & 17.8 & $\mathrm{~N}$ & $\ldots$ & $\ldots$ & \\
\hline $\mathrm{B} 2215+020$ & 3.550 & 21.5 & $\mathrm{~N}$ & $\ldots$ & $\ldots$ & \\
\hline B2224+006 & 2.248 & 21.7 & $\mathrm{~N}$ & $\ldots$ & $\ldots$ & \\
\hline B2245-059 & 3.295 & 19.5 & $\mathrm{~N}$ & $\ldots$ & $\ldots$ & \\
\hline B2245-328 & 2.268 & 16.5 & $\mathrm{~N}$ & $\ldots$ & $\ldots$ & \\
\hline B2256+017 & 2.663 & 19.0 & $\mathrm{~N}$ & $\ldots$ & $\ldots$ & 8 \\
\hline B2311-373 & 2.476 & 18.5 & $\mathrm{Y}$ & 3.0 & 2.182 & \\
\hline B2314-340 & 3.100 & 18.5 & $\mathrm{~N}$ & $\ldots$ & $\ldots$ & \\
\hline \multirow[t]{2}{*}{ B2314-409 } & 2.448 & 18.0 & $\mathrm{Y}$ & 4.0 & 1.857 & \\
\hline & & & $\mathrm{Y}$ & 2.0 & 1.875 & \\
\hline B2315-172 & 2.462 & 19.5 & $\mathrm{~N}$ & $\ldots$ & $\ldots$ & \\
\hline B2325-150 & 2.465 & 19.5 & $\mathrm{~N}$ & $\ldots$ & $\ldots$ & \\
\hline B2351-154 & 2.665 & 19.0 & $\mathrm{~N}$ & $\ldots$ & $\ldots$ & \\
\hline
\end{tabular}

we also took spectra using R600 grism with the similar spectral resolution to cover 4320-6360 A. The seeing conditions remained around 1.2 arcsec, although the sky was not photometric during all four nights. However, since we normalise our spectra to fit the DLA profile, the accurate flux calibration is not critical for the purpose of this paper.

\subsection{AAT observations}

The RGO spectrograph was used with the TEK CCD, $25 \mathrm{~cm}$ camera and $600 \mathrm{~V}$ grating. The seeing at Siding Spring was highly variable through these observations, ranging from 0.8 to 2.0 arcsecs, often with large fluctuations during a given night. However, the slit width was fixed at 1.5 arcsec which projected onto 1.8 pixels to give a $F W H M$ resolution of $3 \AA$. The grating angle was chosen to cover the entire wavelength range of each QSO from at least $3400 \AA$ (corresponding to the wavelength of Lyman $\alpha \lambda 1216$ at $\left.z_{\text {abs }}=1.8\right)$ to $z_{\mathrm{em}}$. The grating angle varied slightly for each run depending on the QSO subset to be observed, but was typically around $19.74^{\circ}$, corresponding to a central wavelength of $\lambda=4000 \AA$. At this setting, the wavelength range is $\sim 3200 \AA-4800 \AA$. For the few bright, high redshift $\left(z_{\mathrm{em}} \gtrsim 2.9\right)$ targets observed with the AAT, a second grating setting, with a central wavelength $\lambda=5500 \AA$, was required to cover the spectrum up to $6300 \AA$.

\subsection{VLT observations}

The VLT observations were executed with FORS1 in a combination of service and visitor mode. The 600B grating was used for all targets, with additional wavelength coverage provided by the $600 \mathrm{R}$ grating for the $z_{\mathrm{em}}=4.458$ QSO B1251-407. A slit width of 0.7 arcsec provided a FWHM resolution of $4.5 \AA$ and a wavelength coverage of 3360-5760 $\AA$. The $600 \mathrm{R}$ grating gave additional coverage (only required for the highest redshift QSO in our sample) over $5200-7300 \AA$ with a resolution of $3.6 \AA$. Despite the faintness of several of the VLT targets (down to $B=24$ ), all acquisitions could be executed in "fast" mode and without blind offsets.

\subsection{Data reduction and column density fitting}

We applied the same reduction procedure to all of the data. The standard IRAF $^{2}$ routines were used. First, all of the images were trimmed and the bias level was subtracted using the over-scan regions. High $S / N$ flat-field images were combined to a single image, which was then smoothed using a box median filter of $1 \times 50$ pixels. The orientation of the box median filter is chosen such that we preserve all of the variations along the dispersion axis. Then, the normalized 2-D flat-field image was used to remove pixel-to-pixel variations in the quasar images. The task APALL was used to perform the optimal extraction of the 1-D spectra. Wavelength calibration images were typically taken both before and after each QSO image. The comparison lamps are $\mathrm{CuAr}$ at AAT, NeHe at ESO $3.6 \mathrm{~m}$ and $\mathrm{NeHgCd}$ on FORS1 at VLT. The dispersion solutions were obtained using a 4th order Legendre polynomial and the RMS error of the fitting was less than $0.05 \AA$.

\footnotetext{
${ }^{2}$ IRAF is distributed by the National Optical Astronomy Observatories, which are operated by the Association of Universities for Research in Astronomy, Inc, under cooperative agreement with the National Science Foundation.
} 
Finally, we performed error-weighted summation of all of the wavelength calibrated spectra for each QSO. The final error array was the quadratic sum of the individual error spectra.

The spectra of all 58 QSOs observed by us are presented in Fig. 1. Once extracted, the spectra were inspected for the presence of DLAs - the Lyman $\alpha$ signature clearly visible as a broad, saturated absorption feature. If a DLA was identified, the spectrum was normalised by dividing through by the QSO continuum and then fitted with a Lyman $\alpha$ profile using the Starlink package DIPSO to determine the redshift and column density of the DLA. The complete list of CORALS QSOs and identified DLAs can be found in Table 3 .

\section{Damped Lyman alpha systems in the CORALS survey}

We adopt the usual definition of a DLA, i.e. $N(\mathrm{H} \mathrm{I}) \geq$ $2 \times 10^{20} \mathrm{~cm}^{-2}$, although our data are of sufficient resolution and $S / N$ to recognise and measure absorbers of somewhat lower $N(\mathrm{H} \mathrm{I})$ (Ellison 2000). From the observations of the 66 CORALS QSOs, a total of 22 DLAs have been identified. Three of these have absorption redshifts similar to the emission redshift of the QSO. We follow the standard procedure of excluding DLAs within $3000 \mathrm{~km} \mathrm{~s}^{-1}$ of the QSO redshift from our statistical analysis, in order to facilitate comparison with other surveys. However, we note that the $z_{\mathrm{abs}} \sim z_{\mathrm{em}}$ DLAs are probably similar to intervening absorbers (Møller et al. 1998) and we defer the analysis of the "associated" CORALS DLAs to a future paper (Ellison et al. in preparation). Of the 19 intervening DLAs, two (B0458-020 and B0528-250a) were already known and have been extensively studied prior to our survey. Profile fits to all the others are shown in Fig. 2. We now briefly discuss each DLA system.

B0335-122 Extended absorption around the DLA trough complicates the fitting of this system. The O I $\lambda 1302$ line is used to constrain redshift, but the error on the final fit is relatively high $\left(6 \pm 1 \times 10^{20} \mathrm{~cm}^{-2}\right)$ due the critical, yet uncertain, continuum placement.

B0347-211 The combination of low absorption redshift and faint QSO magnitude have resulted in a low $S / N$ spectrum of this DLA and a relatively poor fit. The redshift is constrained by the $\mathrm{Al}$ II $\lambda 1670$ line and the $N(\mathrm{H} \mathrm{I})$ determined by fitting a damped profile is in good agreement with the column density inferred from the equivalent width measurement.

B0432-440 Although the spectrum is noisy, the column density of this DLA is reasonably constrained by the base of its trough and the shape of its red wing. This system is well-fitted with a column density $N(\mathrm{H} \mathrm{I})=6 \times 10^{20} \mathrm{~cm}^{-2}$.
B0438-436 Both AAT and VLT spectra were obtained for this QSO. The best fit to the combined spectrum has $N(\mathrm{H} \mathrm{I})=6 \times 10^{20} \mathrm{~cm}^{-2}$, although this is constrained mostly by the red wing since there is significant contaminating absorption in the blue wing. The redshift of the $\mathrm{Al}$ II $\lambda 1670$ line (with rest-frame equivalent width $W_{0}=0.63 \AA$ ) agrees well with the redshift determined from the centre of the DLA trough.

B0537-286 A relatively high $S / N$ spectrum and simple structure around the absorber permit a good fit to this DLA.

B0913+003 An excellent fit to this DLA is facilitated by the clearly defined damping wings and lack of blending.

B0933-333 Despite blending with a weaker component blueward of the DLA, this system is reasonably fit with profile of $N(\mathrm{H} \mathrm{I})=3 \times 10^{20} \mathrm{~cm}^{-2}$.

B1055-301 This DLA has a very large equivalent width $\left(W_{0} \sim 60 \AA\right.$ ) but is heavily blended with other absorption lines. The fit is only constrained by the base of the absorption which is clearly saturated over $10 \AA$ in the rest frame. Higher order Lyman lines are not available to improve the decomposition of the H I cloud model, although several metal transitions are covered by the AAT spectrum. Strong Si II $\lambda 1526, \mathrm{Al}$ II $\lambda 1671$ and Fe II $\lambda 1608$ are all observed with redshifts of 1.9037 and rest frame equivalent widths $W_{0}=1.2 \AA, 1.3 \AA$ and $0.8 \AA$ respectively. This provides strong support for the presence of a DLA at the position shown in Fig. 2. However, the column density can only be constrained to within $\pm 15 \%$ : $N(\mathrm{H} \mathrm{I})=$ $35 \pm 5 \times 10^{20} \mathrm{~cm}^{-2}$.

B1228-113 Another low redshift system whose spectrum has a low $S / N$, although the lack of strong nearby Lyman $\alpha$ forest lines results in an acceptable fit.

B1230-101 Constrained mostly by its fit to the red wing, this DLA at $z_{\mathrm{abs}}=1.931$ has several metal lines associated with it. The AAT spectrum covers both Fe II $\lambda 1608\left(W_{0}=\right.$ $520 \mathrm{~m} \AA)$ and Si II $\lambda 1526\left(W_{0}=720 \mathrm{~m} \AA\right)$; the latter lies just blueward of a strong, resolved, C IV doublet at $z_{\text {abs }}=$ 1.899. There is a second C IV system at $\lambda_{\text {obs }} \sim 4540 \AA$ associated with the DLA itself.

B1251-407a,b As can be seen in Fig. 1, this QSO has two prominent absorption lines at approximately 5510 and $5780 \AA$, corresponding to Lyman $\alpha$ at $z_{\text {abs }}=3.533$ and 3.752 respectively. The former is well reproduced by a damped profile with $N(\mathrm{H} \mathrm{I})=4 \times 10^{20} \mathrm{~cm}^{-2}$. The latter has a large equivalent width $\left(W_{0}=13.7 \AA\right)$, but also steep sides and cannot be fitted satisfactorily with a single absorption component. Closer inspection reveals structure 


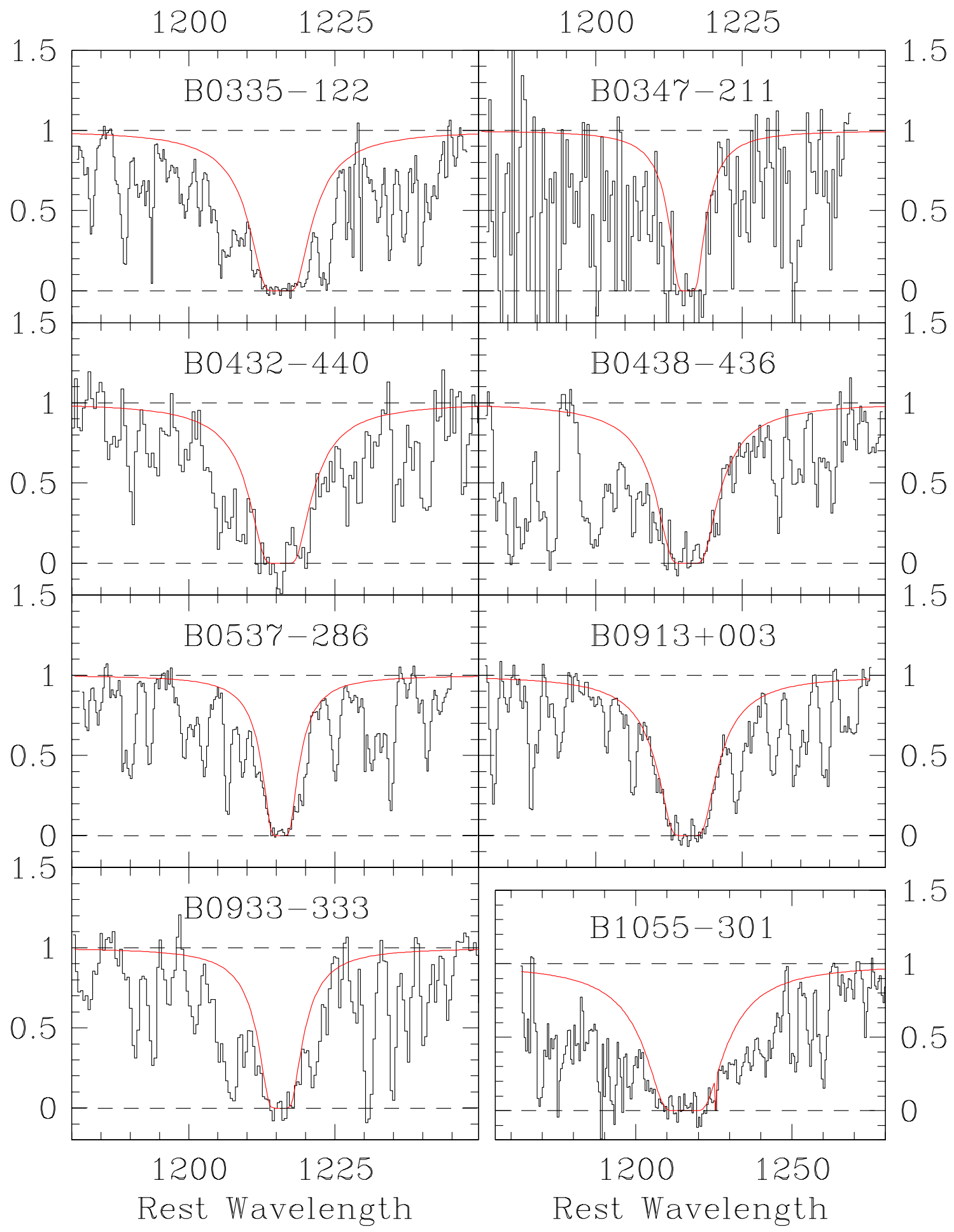

Fig. 2. Damped Lyman $\alpha$ profile fits (continuous lines) to all newly discovered intervening DLAs. See Table 3 for the values of $N(\mathrm{H} \mathrm{I})$ and $z_{\mathrm{abs}}$ corresponding to the theoretical profiles shown. Note that the bottom right-hand panel is on a different wavelength scale due to the large column density of the DLA towards B1055-301.

within the core of the damped Lyman $\alpha$ line (see Fig. 2). We consider this feature to be a composite consisting of a DLA at $z_{\text {abs }}=3.752$ with $N(\mathrm{H} \mathrm{I})=2 \times 10^{20} \mathrm{~cm}^{-2}$ flanked by two lower column density components. This interpretation is supported by the presence of Si II $\lambda 1526$ absorption at the same redshift as the DLA. In any case, both DLAs in this QSO are excluded from our discussion of the sample statistics below because we consider only the redshift interval $1.8<z_{\text {abs }}<3.5$ (see Sect. 6). The two other moderately large $E W$ systems at $\lambda=4640$ and $4830 \AA$ respectively (see Fig. 1), are not DLAs, but blends of lower column density lines. 


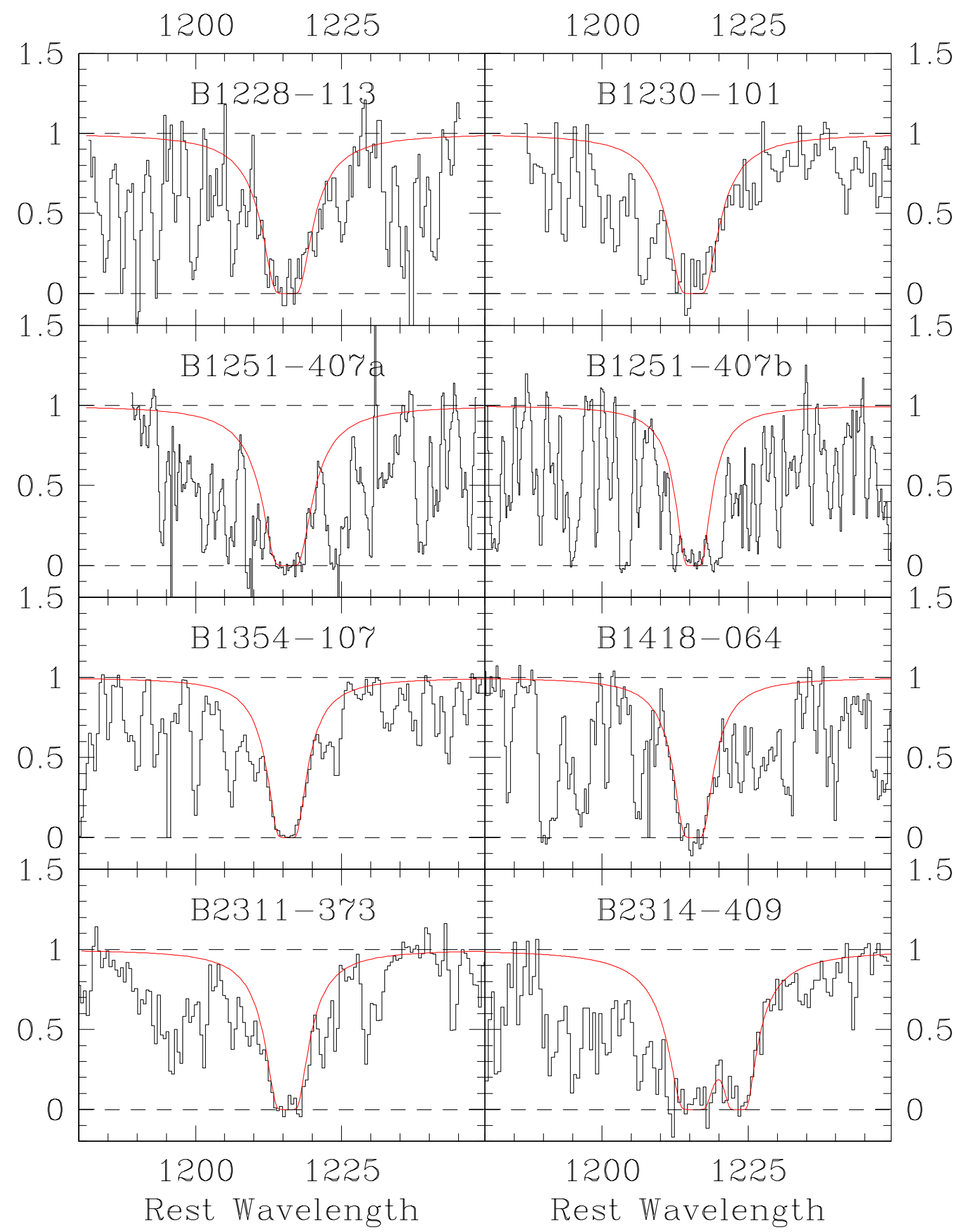

Fig. 2. continued.

B1354-107a Two strong absorption features are seen towards this QSO. Absorber "a" has a redshift of $z_{\mathrm{abs}}=$ 2.501 and is well fitted by a damped profile with $N(\mathrm{H} \mathrm{I})=$ $2.5 \times 10^{20} \mathrm{~cm}^{-2}$. Absorber "b", with $N(\mathrm{H} \mathrm{I})=6 \times$ $10^{20} \mathrm{~cm}^{-2}$ and $z_{\text {abs }}=2.966$, is classified as a $z_{\text {abs }} \sim z_{\mathrm{em}}$ DLA and is not included in the present analysis.
B1418-064 At $z_{\mathrm{abs}}=3.449$, this is the highest redshift DLA to be included in our statistical analysis; the Lyman $\alpha$ line is well reproduced by a damped profile with $N(\mathrm{H} \mathrm{I})=2.5 \times 10^{20} \mathrm{~cm}^{-2}$. Our limited spectral coverage redward of Lyman $\alpha$ emission reveals associated Si II $\lambda 1304$ and O I $\lambda 1302$ absorption lines with $W_{0}=290$ and $390 \mathrm{~m} \AA$ respectively (although the O I transition is 
probably blended with an unidentified line and the equivalent width measurement is therefore an overestimate).

B2311-373 A column density of $N(\mathrm{H} \mathrm{I})=3 \times 10^{20} \mathrm{~cm}^{-2}$ provides a satisfactory fit to the base and both wings of this DLA. Si II $\lambda 1526$ is the only metal transition identified in the AAT spectrum with $W_{0}=500 \mathrm{~m} \AA$.

B2314-409a,b As is the case for B1055-301, there is extended $\mathrm{H}$ I absorption in this spectrum. The spike at $\lambda_{0} \sim 1220 \AA$ in Fig. 2 could be either noise or residual continuum flux between two closely spaced components. The latter interpretation is supported by the presence of two Si II $\lambda 1526$ absorption lines at redshifts $z_{\text {abs }}=1.857$ and 1.875 respectively. These redshifts match well the blue and red components of the Lyman $\alpha$ absorption feature, as indicated by the fit shown in Fig. 2. The metal lines are stronger in the $z_{\text {abs }}=1.857$ system, where we also detect Fe II $\lambda 1608$ and Al II $\lambda 1671$; the $z_{\text {abs }}=1.875$ component may well be a very low metallicity system given that Fe II $\lambda 1608$ has $W_{0} \leq 90 \mathrm{~m} \AA$. This is the second case of a multiple DLA, the first being a triple DLA (CTQ247) spread over $\sim 6000 \mathrm{~km} \mathrm{~s}^{-1}$, discovered by Lopez et al. (2000).

\section{DLA statistics-number density and $\Omega_{\text {DLA }}$}

One of the key areas of interest in the study of DLAs is how the population evolves with time. If DLAs represent the bulk of the galaxy population at a given epoch, then studying their properties as a function of redshift will be a powerful method for tracing galaxy evolution. For example, there is strong evidence for redshift evolution in the column density distribution function, $f(N)$ (StorrieLombardi \& Wolfe 2000). However, since the number density is well represented by a power law there appears to be no change in the product of space density and absorber cross section. As discussed in the Introduction, the evolution of $\Omega_{\mathrm{DLA}}$ is still unclear, particularly at $z_{\mathrm{abs}}<1.5$ where statistics are poor.

Due to the relatively small size of the CORALS survey, it is not possible to investigate the evolution of DLA statistics for this sample. However, since our main objective is to ascertain whether or not a significant fraction of gas has gone undetected, it is sufficient for us to restrict our determination of $\Omega_{\mathrm{DLA}}$ to the range $1.8<z_{\text {abs }}<3.5$ where there appears to be little evolution. This requires us to omit two DLAs from our sample (B1251-407a and b), and restrict our statistical analysis to the remaining 17 DLAs.

\subsection{DLA number density}

This is simply the total number of DLAs divided by the total intervening redshift interval covered, $\Delta z$, given by

$\Delta z=\sum_{i=1}^{n}\left(z_{i}^{\max }-z_{i}^{\min }\right)$ where the summation is over the $n$ QSOs in the sample. For CORALS, $z_{\min }=1.8^{3}$ and $z_{\max }=3.5$ or $z_{3000}$ (the redshift corresponding to a $v=3000 \mathrm{~km} \mathrm{~s}^{-1}$ ), whichever is smaller:

$z_{3000}=\left[\left(z_{\mathrm{em}}+1\right) \sqrt{\frac{(b-1)}{(-b-1)}}\right]-1$

and

$b=\frac{v}{c}=0.01$.

In cases where a Lyman limit system is present in the QSO spectrum within the redshift range considered,

$z_{\min }=\frac{\left(z_{\mathrm{LLS}}+1\right) \times 912}{1216}-1$

The total redshift interval for the CORALS sample is $\Delta z=55.46$ within which we detect 17 DLAs. Thus, $n(z)=0.31_{-0.08}^{+0.09}$ at a mean absorption redshift $\left\langle z_{\text {abs }}\right\rangle=$ 2.37 (the errors were calculated using the prescription by Gehrels 1986 for small number statistics).

Taken at face value, the estimate of $n(z)$ from the CORALS survey is $\sim 50 \%$ larger (at the same $\left\langle z_{\text {abs }}\right\rangle$ ) than that found in previous surveys. For example, StorrieLombardi \& Wolfe (2000) deduced $n(z)=0.055(1+$ $z)^{1.11}=0.21$ (no errors quoted). However, this difference is only marginally significant, since the two determinations of $n(z)$ are within $\sim 1 \sigma$ of each other.

\subsection{The mass density of neutral gas in DLAs}

The mass density of neutral gas in DLAs as a fraction of the closure density is expressed as

$\Omega_{\text {DLA }}=\frac{H_{0} \mu m_{\mathrm{H}}}{c \rho_{\text {crit }}} \int_{N_{\min }}^{N_{\max }} N f(N) \mathrm{d} N$

where $\mu$ is the mean molecular weight $(=1.3), m_{\mathrm{H}}$ is the mass of a hydrogen atom, and $f(N)$ is the column density distribution. We avoid making a priori assumptions about the functional shape of $f(N)$ by adopting the approximation by Storrie-Lombardi et al. (1996)

$\int_{N_{\min }}^{N_{\max }} N f(N, z) \mathrm{d} N=\frac{\sum_{i} N_{i}(\mathrm{H} \mathrm{I})}{\Delta X}$

so that

$\Omega_{\mathrm{DLA}}=\frac{H_{0} \mu m_{\mathrm{H}}}{c \rho_{\mathrm{crit}}} \frac{\sum_{i} N_{i}(\mathrm{H} \mathrm{I})}{\Delta X}$

from which the mass density of neutral gas traced by DLAs is obtained by direct summation of the individual values of neutral hydrogen column density. The redshift path, $\Delta X$,

${ }^{3}$ We note that although most surveys calculate $z_{\min }$ for each QSO on a case by case basis, depending on wavelength coverage and $S / N$, we have designed the CORALS survey to have adequate spectral range and $S / N$ to achieve a uniform lower redshift cut-off. 


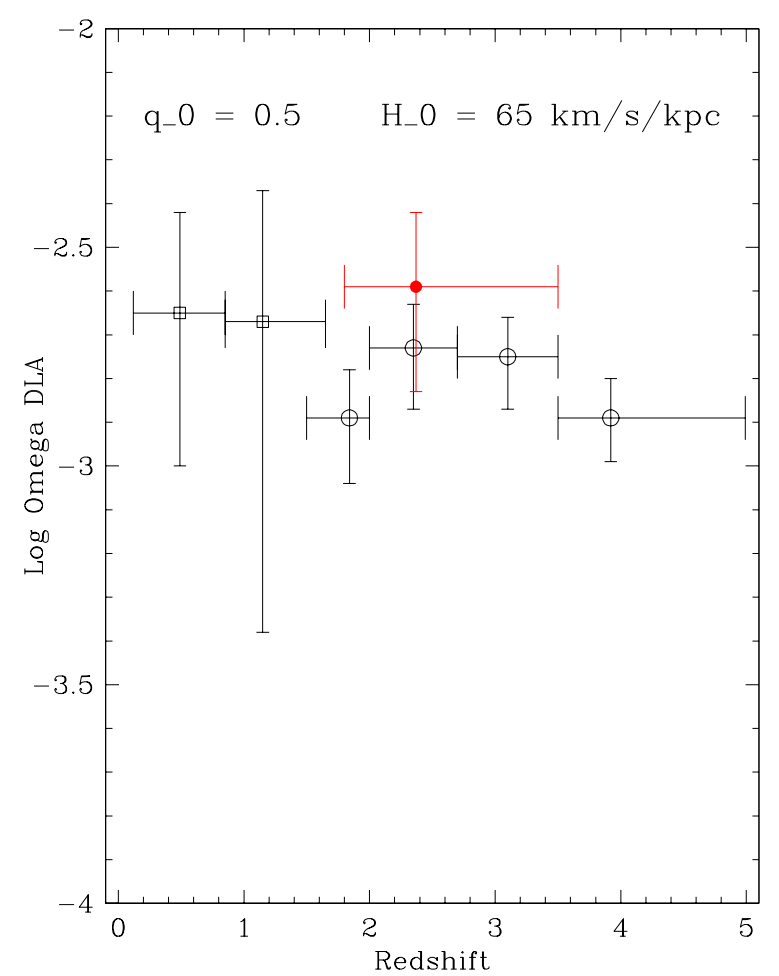

Fig. 3. The mass density of neutral gas, $\Omega_{\mathrm{DLA}}$, in DLAs. Open circles and squares are measurements from the latest compilations by Péroux et al. (2001b) and Rao \& Turnshek (2000) respectively. The solid circle is the value from the CORALS survey presented here for the redshift interval $1.8<z_{\text {abs }}<3.5$.

which takes into account co-moving distances, is given (in our adopted cosmology) by

$\Delta X=\sum_{i} \frac{2}{3}\left[\left(1+z_{\max , i}\right)^{\frac{3}{2}}-1\right]-\frac{2}{3}\left[\left(1+z_{\min , i}\right)^{\frac{3}{2}}-1\right]$.

From Eqs. (7) and (8) with the values of $N(\mathrm{H} \mathrm{I})$ listed in Table 3 we deduce $\log \Omega_{\text {DLA }} h=-2.59_{-0.24}^{+0.17}$ (with errors calculated as described by Storrie-Lombardi et al. 1996). As can be seen from Fig. 3, we again find that the CORALS value of $\Omega_{\mathrm{DLA}}$ is in good agreement with previous determinations. Thus, the most straight-forward conclusion from the CORALS survey is that existing magnitude limited samples of QSOs do not seriously underestimate the number of DLAs, nor their overall mass content; by and large, they seem to provide a fair census of neutral gas at high redshift. On the other hand, given the current statistical uncertainties, the data still admit a moderate degree of dust bias. The $1 \sigma$ limits on both $n(z)$ and $\Omega_{\text {DLA }}$ include the possibility that analyses based on optically selected samples may have underestimated both quantities by a factor of $\sim 2$. This possibility is further highlighted when we consider the distribution of values of $N(\mathrm{H} \mathrm{I})$ in the CORALS survey (Fig. 4). Given the small size of our survey, we may well be missing DLAs at the high column density end of the distribution simply through small number statistics. We return to this point in Sect. 6.3 below. We note that although it is impossible to constrain the $\mathrm{H}$ I column density

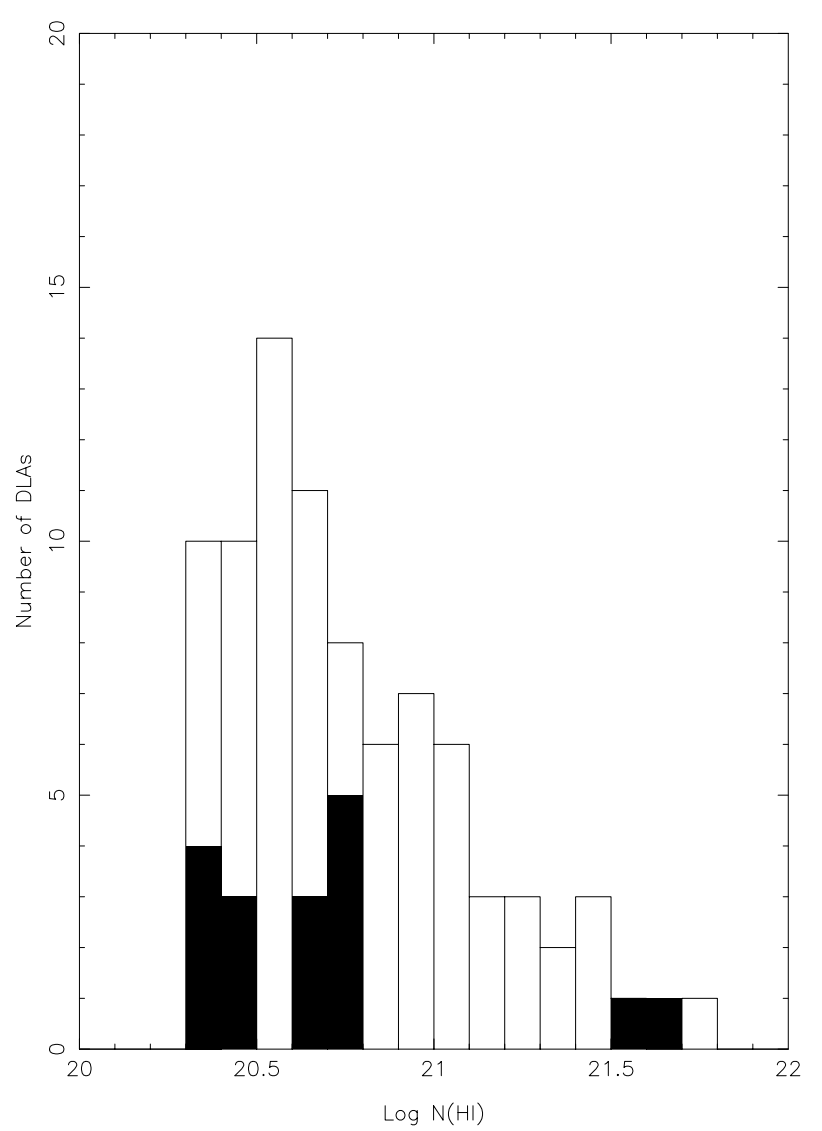

Fig. 4. Distribution of H I column densities in the large sample of Storrie-Lombardi \& Wolfe (2000) and in the CORALS survey (shaded histogram). Due to the relatively small number of QSOs, and therefore DLAs, included in CORALS, we do not fully sample the $N(\mathrm{H} \mathrm{I})$ distribution.

distribution for the CORALS sample, we do detect 2 relatively rare high $N(\mathrm{H} \mathrm{I})$ systems. Therefore, although we do not find evidence for a previously excluded population of very high column density absorbers, with improved statistics it would be of great interest to re-assess the $N(\mathrm{H} \mathrm{I})$ distribution function.

We also consider another effect. As explained in Sect. 2, many previous surveys have included in their statistical analyses candidate DLAs, identified on the basis of the equivalent width of the Lyman $\alpha$ line rather than by profile fitting to a damped profile. It is worthwhile examining the overestimate of $\Omega_{\mathrm{DLA}}$ which may result from this approximation. For the 17 DLAs for which we have obtained our own spectra, we measure the equivalent width and compare the implied column density to that determined by fitting the Lyman $\alpha$ line. In most cases we find the two techniques to be in very good agreement, certainly within the errors associated with each method. There are only three exceptions (the DLAs in B1055-301, B1251-407a and B2314-409) where the equivalent width determination leads to a much higher $N(\mathrm{H} \mathrm{I})$ than the line fit. Inspection of Fig. 2 shows that this is due to extended absorption around the DLA. Although fits of these DLAs were not straight-forward, this process was 
facilitated by higher spectral resolution and coverage of metal lines which provide additional guidance in the shape of the wings and $z_{\text {abs }}$. Had we used the values of $N(\mathrm{H} \mathrm{I})$ deduced from the equivalent widths for the entire CORALS sample, we would have over-estimated $\Omega_{\text {DLA }}$ by $20 \%$. This discrepancy would have been further increased if extended blends of lines which do not include a DLA, such as those present in the spectrum of B1251-407, were mistakenly included. Nevertheless, the over-estimate is not large and, given the increasing body of accurate measurements of $N(\mathrm{H} \mathrm{I})$ in DLAs, we think it very unlikely that this effect could be masking a higher degree of dust bias than that indicated by inspection of Fig. 3.

Finally, for completeness, we calculate $\Omega_{\mathrm{DLA}}$ in the redshift range $3.5<z_{\text {abs }}<4.0$ where we detect two DLAs despite the fact that with CORALS we only sample a total interval $\Delta z=1.26$. The error bars are naturally very large, but all the same it is intriguing that $\log \Omega_{\text {DLA }} h$ seems to remain high at $-2.37_{-0.59}^{+0.24}$ in contrast with the slight down-turn suggested by the work of Storrie-Lombardi \& Wolfe (2000) and Péroux et al. (2001b). It will be very interesting to see how better statistics will impact upon the CORALS value of $\Omega_{\mathrm{DLA}}$ at the highest redshifts since the present determination would suggest an increase in the importance of dust bias with increasing redshift.

\subsection{DLA statistics as a function of B-band magnitude}

In their preliminary analysis of this sample, Ellison et al. (2000) found tentative evidence that $\Omega_{\text {DLA }}$ was higher towards fainter QSOs, consistent with the effect expected from a dust bias. We re-examine this point in Fig. 5, which shows cumulative statistics for the CORALS DLAs as a function of the $B$-band magnitude of the background QSOs. Since there are relatively few bright QSOs in our sample, we also show the statistics for the LBQS DLA survey (Wolfe et al. 1995) which has a limit $B \lesssim 19$, using the column densities reported by Wolfe et al. (1995) and Storrie-Lombardi \& Wolfe (2000). We confirm the initial conclusion by Ellison et al. (2000) that $\Omega_{\text {DLA }}$ increases as fainter QSOs are observed, but stabilises at $B \simeq 20.0$. Thus, we do not find a population of high $N(\mathrm{H} \mathrm{I})$ DLAs which is only revealed when faint $(B \gtrsim 20)$ QSOs are observed. However, closer inspection of the data emphasises the need to extend our survey in order to fully sample the column density distribution function, particularly at the high column density end. The increase in $\Omega_{\text {DLA }}$ between the $B \leq 19.0$ and $B \leq 19.5$ bins is almost entirely due to a single DLA with $N(\mathrm{H} \mathrm{I})=3.5 \times 10^{21} \mathrm{~cm}^{-2}$. Similarly, the increase between the $B \leq 19.5$ and $B \leq 20.0$ bins is caused by a single DLA with $N(\mathrm{H} \mathrm{I})=4.5 \times 10^{21} \mathrm{~cm}^{-2}$. Between them, these two systems account for over half of the neutral gas in the entire DLA sample. A K-S test that compares the distribution of $N(\mathrm{H} \mathrm{I})$ among DLAs towards $B<20$ QSOs with those from the large sample of StorrieLombardi \& Wolfe (2000) provides inconclusive results,
All 21.020 .520 .019 .519 .0

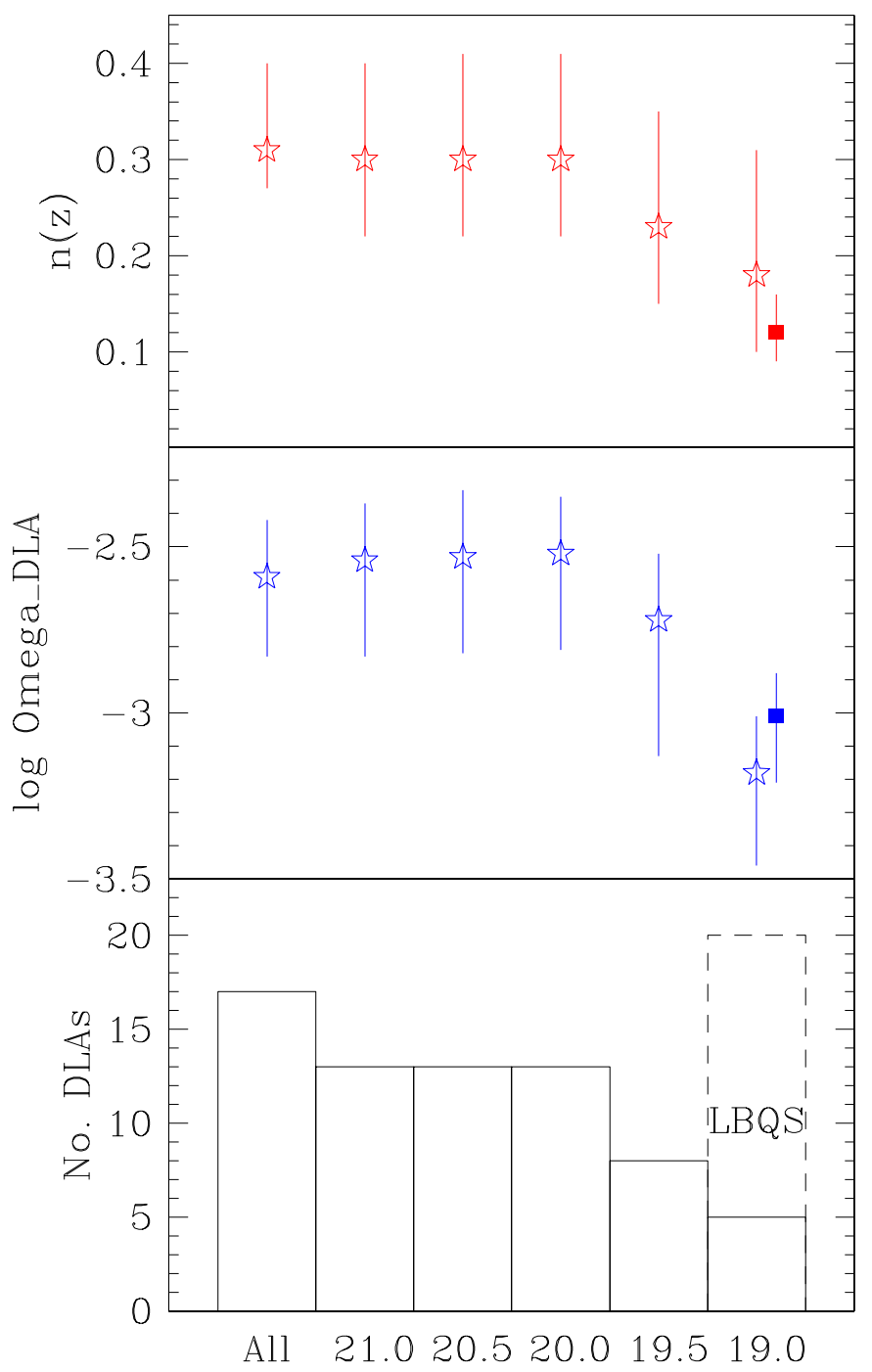

Fig. 5. DLA statistics as a function of magnitude. The quantities plotted are for the cumulative values in the bins $\leq$ the $B$-band magnitude on the $x$-axis. Bottom panel: cumulative histogram of number of DLAs. The dashed area shows the number of LBQS DLAs used to determine improved statistics towards bright QSOs. Middle and top panels: stars show the values for CORALS DLAs, whilst the solid square shows the same quantity for the LBQS sample $(B \lesssim 19)$; this point has been offset on the $x$-axis for clarity).

i.e. that the samples are indistinguishable at only the $1 \sigma$ level. Taken as a whole, the CORALS DLAs are inconsistent (again only at the $1 \sigma$ level) with the $N(\mathrm{H} \mathrm{I})$ distribution of Storrie-Lombardi \& Wolfe (2000). As found by Ellison et al. (2000) for a sub-sample of the CORALS sample, we confirm that for the complete sample that there is no strong correlation between magnitude and redshift out to $z \sim 3.5$. Therefore, these trends are not likely to be associated with the evolution (apparent or real) of the properties of the QSOs themselves.

In addition to the cumulative values of $n(z)$ shown in Fig. 5, we calculate the number density of DLAs towards 
QSOs with $B \geq 20$ and $B<20$, and find $n(z)=0.38_{-0.14}^{+0.20}$ $\left(\right.$ at $\left.\left\langle z_{\text {abs }}\right\rangle=2.54\right)$ and $0.27_{-0.08}^{+0.11}\left(\right.$ at $\left.\left\langle z_{\text {abs }}\right\rangle=2.25\right)$ respectively. For the $B<20$ subset, this value is consistent with the number density found by Storrie-Lombardi \& Wolfe (2000). Again, we see that there is an excess of DLAs in faint QSOs, but only at the $\sim 1 \sigma$ significance level.

\section{Summary and discussion}

We have presented the first results from the CORALS survey for DLAs in a radio-selected sample of QSOs. The sample consists of $66 z_{\mathrm{em}} \geq 2.2$ QSOs, 58 of which have been observed by us using the ESO $3.6 \mathrm{~m}$, the AAT and the VLT facilities, while the remaining eight were culled from the literature. All the new spectra are presented in Fig. 1. A total of 22 DLAs has been identified, 19 of which are at intervening redshifts.

We find that the comoving mass density of neutral gas implied by these DLAs is $\log \Omega_{\mathrm{DLA}} h=-2.59_{-0.24}^{+0.17}$ at a mean $\left\langle z_{\text {abs }}\right\rangle=2.37$, in good agreement with previous surveys. Similarly, the number density of DLAs per unit redshift in radio-selected QSOs, $n(z)=0.31_{-0.08}^{+0.09}$, is only $\sim 1 \sigma$ higher than that in optical, magnitude-limited samples. Within our own sample we also find that $n(z)$ is higher, but again by only $\sim 1 \sigma$, in QSOs with $B>20$, compared with sightlines towards brighter quasars. These results indicate that at redshifts $z=2-3.5$ DLA surveys using optically selected QSOs probably underestimate the number of DLAs, and the gas mass they trace, by no more than a factor of about 2, in broad agreement with the predictions by Pei \& Fall (1995). In particular, we have not uncovered a population of high column density $N(\mathrm{H} \mathrm{I})>10^{21} \mathrm{~cm}^{-2}$ absorbers which had been missed in previous searches limited to QSOs brighter than $B \simeq 20$.

These conclusions are somewhat tentative because of the small size of the CORALS sample. Our value of $\Omega_{\text {DLA }}$ is dominated by two very high column density systems, both of which occur in moderately bright $(B=19.5,20)$ QSOs, and the column density distribution function of DLAs is clearly not well sampled with the relatively small number of QSOs in our survey.

The next important step in this work is to determine the metallicities and dust content of CORALS DLAs. The possibility of a dust bias in DLA selection has previously been appealed to in order to explain the lack of high column density, metal-rich absorbers (e.g. Prantzos \& Boissier 1999). This explanation now seems less likely, regardless of whether or not CORALS DLAs prove to be more metal-rich, simply because high column density DLAs do not appear to be significantly more common in fainter QSOs, at least within the statistical limitations of our survey. Nonetheless, determining the metallicities of the new DLAs discovered here remains an important goal, because it will allow us to assess whether the low element abundances found so far are indeed typical of the full DLA population.

What could then be the reason for the observed dearth of high column density, metal-rich DLAs? We consider it unlikely that gravitational lensing may be the answer. In principle one may conjecture that close alignment of QSOs with foreground galaxies may produce a tendency for such sightlines to be deflected away from the inner regions of galaxies, where interstellar clouds with high $N(\mathrm{H} \mathrm{I})$ and high $Z$ may be preferentially intercepted. However, to date no statistical evidence for lensing of QSOs by DLAs has been found (Le Brun et al. 2000) and, in any case, lensing would be most effective at significantly lower redshifts than those considered here (Smette et al. 1997).

A more plausible explanation is that there is simply a cross-sectional bias against detecting DLAs in sightlines that pass through the centres of galaxies. Observationally, one could argue that high $N(\mathrm{H} \mathrm{I})$, high $Z$ DLAs have already been found in the Lyman break galaxies (e.g. Pettini et al. 2000) which indeed have typical linear sizes one order of magnitude smaller than the impact parameters of most DLAs (Giavalisco et al. 1996; Calzetti \& Giavalisco 2000; see also Fig. 1 of Pettini 2001). Theoretical studies of DLAs also support this interpretation. For example, the models of Mathlin et al. (2001), who simulated DLA surveys by sampling model galaxies at random impact parameters, predict that the locus of high column density and metal rich absorbers should be populated, but that DLAs with these properties are intrinsically rare due to the small cross-sectional area presented by the inner galactic regions where they are found.

As already emphasised, the CORALS data set is too small to sample properly the column density distribution function and a considerably larger survey is required in order to provide the statistical coverage that will determine the true incidence of $N(\mathrm{H} \mathrm{I})>10^{21} \mathrm{~cm}^{-2}$ absorbers. A statistically larger survey will not only improve our determinations of $\Omega_{\mathrm{DLA}}$ and $n(z)$, but will also make it possible to investigate the evolution (or lack thereof) of these quantities with redshift. It will be particularly interesting to examine the possibility of higher dust bias at larger redshifts as suggested by the data in Fig. 3; such data will offer an insight into the evolution of dust at early epochs. One promising prospect for extending the work presented here is the FIRST QSO survey (Gregg et al. 1996; White et al. 2000). Although spectral follow-up has so far been limited to bright $R<19$ targets, future followup of FIRST sources at fainter optical magnitudes would provide an excellent complement to the CORALS survey.

Acknowledgements. It is a pleasure to acknowledge the consistent support of this project by the ESO and AAT Time Assignment Panels and the professional and efficient help of the telescope staff at the AAT, ESO 3.6-m and VLT. In particular, we are grateful to the ESO Paranal science operations staff for their expert execution of our service observations. We thank Mauro Giavalisco and Lisa Storrie-Lombardi for obtaining spectra of two of our targets and Joop Schaye for useful comments on an earlier draft of this paper. 


\section{References}

Boissé, P., Le Brun, V., Bergeron, J., \& Deharveng, J.-M. 1998, A\&A, 333, 841

Calzetti, D., \& Giavalisco, M. 2000, Ap. \& Sp. Sci., in press [astro-ph/0012068]

Carilli, C. L., Menten, K. M., Reid, M. J., Rupen, M. P., \& Yun, M., 1998, ApJ, 494, 175

Churchill, C. W. 2001, ApJ, submitted [astro-ph/0105044]

Dessauges-Zavadsky, M., D'Odorico, S., McMahon, R. G., et al. 2001, A\&A, 370, 426

Drinkwater, M. J., Barnes, D. G., \& Ellison, S. L. 1995, PASA, 12,248

Ellison, S. L. 2000, Ph.D. Thesis (University of Cambridge)

Ellison, S. L., Yan, L., Hook, I., et al. 2000, ESO Messenger, 102

Fall, S. M., \& Pei, Y. 1993, ApJ, 402, 479

Fall, S. M., Pei, Y., \& McMahon, R. G. 1989, ApJL, 341, 5

Gehrels, N. 1986, ApJ, 303, 336

Giavalisco, M., Steidel, C. C., \& Macchetto, D. 1996, ApJ, 470, 189

Gregg, M. D., Becker, R. H., White, R. L., et al. 1996, AJ, 112, 407

Hewett, P, Foltz, C., \& Chaffee, F. 1995, AJ, 109, 1498

Kobulnicky, H. A., \& Zaritsky, D. 1999, ApJ, 511, 118

Lanzetta, K. 1993, The Environment and Evolution of Galaxies, ed. Shull \& Thronsen (Dordrecht: Kluwer), 237

Lanzetta, K. M., McMahon, R. G., Wolfe, A. M., et al. 1991, ApJS, 77, 1

Lanzetta, K. M., Wolfe, A. M., \& Turnshek, D. A. 1995, ApJ, 440, 435

Ledoux, C., Petitjean, P., Bergeron, J., Wampler, E. J., \& Srianand, R., 1998, A\&A, 337, 51

Le Brun, V., Smette, A., Surdej, J., \& Claeskens, J.-F. 2000, A\&A, 363, 837

Lopez, S., Maza, J., Masegosa, J., \& Marquez, I. 2000, A\&A, 366,387

Lu, L., Sargent, W. L. W., Barlow, T. A., Churchill, C. W., \& Vogt, S. S. 1996, ApJS, 107, 475

Mathlin, G. P., Baker, A. C., Churches, D. K., \& Edmunds, M. G., 2001, MNRAS, 321, 743

Møller, P., Warren, S. J., \& Fynbo, J. U. 1998, A\&A, 330, 19

Pagel, B. E. J. 2000, in Galaxies in the Young Universe II, ed. H. Hippelein (Berlin: Springer-Verlag), in press [astro-ph/9911204]
Pei, Y., \& Fall, S. M., 1995, ApJ, 454, 69

Pei, Y., Fall, S. M., \& Bechtold, J. 1991, ApJ, 378, 6

Péroux, C., Storrie-Lombardi, L. J., McMahon, R. G., Irwin, M., \& Hook, I. M. 2001a, AJ, 121, 1799

Péroux, C., McMahon, R. G., Storrie-Lombardi, L. J., \& Irwin, M. 2001b, MNRAS, submitted

Pettini, M. 2001, in The Promise of First, ed. G. L. Pilbratt, J. Cernicharo, A. M. Heras, T. Prusti, \& R. Harris, ESA SP-460, in press

Pettini, M., Ellison, S. L., Steidel, C. C., \& Bowen, D. V. 1999, ApJ, 510, 576

Pettini, M., Smith, L. J., King, D. L., \& Hunstead, R.W. 1997, ApJ, 486, 665

Pettini, M., Steidel, C. C., Adelberger, K. L., Dickinson, M., \& \& Giavalisco, M. 2000, ApJ, 528, 96

Prantzos, N., \& Boissier, S. 2000, MNRAS, 315, 82

Prochaska, J. X., \& Wolfe, A. M. 1999, ApJS, 121, 369

Prochaska, J. X., \& Wolfe, A. M. 2000, ApJ, 533, L5

Rao, S. M., \& Turnshek, D. A. 2000, ApJS, 130, 1

Sargent, W., Steidel, C., \& Boksenberg, A. 1989, ApJS, 69, 703

Savaglio, S. 2000, Invited talk at the IAU Symp. 204, The Extragalactic Infrared Background and its Cosmological Implications, Manchester, August 2000, ed. M. Harwit \& M. G. Hauser [astro-ph/0011473]

Shaver, P. A., Wall, J. V., Kellermann, K. I., Jackson, C. A., \& Hawkins, M. R. S. 1996, Nature, 384, 439

Songaila, A. 1998, AJ, 115, 2184

Smette, A., Claeskens, J.-F., \& Surdej, J. 1997, New Astron., 2,53

Storrie-Lombardi, L., Irwin, M., \& McMahon, R. G. 1996, MNRAS, 282, 1330

Storrie-Lombardi, L., McMahon, R. G., \& Irwin, M. 1996, MNRAS, 283, 79

Storrie-Lombardi, L., \& Wolfe, A. M. 2000, ApJ, 543, 552

Tytler, D., Fan, X.-M., \& Burles, S. 1996, Nature, 381, 207

Turnshek, D. A., Wolfe, A. M., Lanzetta, K. M., et al. 1989, ApJ, 344, 567

White, R., et al. 2000, ApJS, 126, 133

Wolfe, A. M. 1988, QSO absorption lines: Probing the universe, Proc. of the QSO Absorption Line Meeting, 296

Wolfe, A. M., Turnshek, D. A., Smith, H. E., \& Cohen, R. D. 1986, ApJS, 61, 249

Wolfe, A. M., Lanzetta, K. M., Foltz, C. B., \& Chaffee, F. H. 1995, ApJ, 454, 698 